\title{
Induced Electromagnetic Fields Estimation in Spine Examinations of MRI Patients: A Re-Evaluation of Existing Clinical Protocols at a Hospital in Accra, Ghana
}

\author{
Frempong Acheampong1,2, Theresa Dery ${ }^{3}$, Rita Appiah", Daniel A. Abaye ${ }^{2 *}$ \\ ${ }^{1}$ Graduate School of Nuclear and Allied Sciences, University of Ghana, Legon, Accra, Ghana \\ ${ }^{2}$ School of Basic and Biomedical Sciences, University of Health and Allied Sciences, Ho, Ghana \\ ${ }^{3}$ Radiological \& Medical Sciences Research Institute, Ghana Atomic Energy Commission, Legon, Accra, Ghana \\ ${ }^{4}$ Ghana Research Rector 1 Center, Ghana Atomic Energy Commission, Legon, Accra, Ghana \\ Email: facheampong@uhas.edu.gh, t.bdery@gaecgh.org, rappiah@gnra.org.gh, ^dabaye@uhas.edu.gh
}

How to cite this paper: Acheampong, F., Dery, T., Appiah, R. and Abaye, D.A. (2018) Induced Electromagnetic Fields Estimation in Spine Examinations of MRI Patients: A Re-Evaluation of Existing Clinical Protocols at a Hospital in Accra, Ghana. Journal of Applied Mathematics and Physics, 6, 1065-1075. https://doi.org/10.4236/jamp.2018.65092

Received: April 24, 2018

Accepted: May 27, 2018

Published: May 30, 2018

Copyright $\odot 2018$ by authors and Scientific Research Publishing Inc. This work is licensed under the Creative Commons Attribution International License (CC BY 4.0).

http://creativecommons.org/licenses/by/4.0/

\section{(c) (i) Open Access}

\begin{abstract}
Patients undergoing Magnetic Resonance Imaging (MRI) are exposed to strong, non-uniform static magnetic fields outside of the central imaging region, in which the movement of the body may induce electric currents in tissues which could possibly be harmful. The purpose of this study was to re-evaluate existing clinical protocols by determining the induced electromagnetic (EM) fields in MRI spine examinations. The study covered 120 MRI spine examinations at the MRI Unit of a hospital in Accra, Ghana. A numerical model based on Faraday's equation was developed using the finite difference method (FDM) and MATLAB software to compute, first, a test simulation of induced EM field intensities and then actual measurements of induced fields on the spine. The simulation results were peak induced electric field, $0.39 \mathrm{~V} / \mathrm{m}$ and current density, $0.039 \mathrm{~A} / \mathrm{m}^{2}$. Patient results were; calculated maximum velocity, $0.29 \mathrm{~m} / \mathrm{s}$; peak induced electric field strength, $0.44 \mathrm{~V} / \mathrm{m}$, and current density, $0.043 \mathrm{~A} / \mathrm{m}^{2}$. The levels of induced EM-fields were such that they would not pose any potential health hazards to the patients as these values were well below the recommended guidance levels set by the Directive IEC 60601-2-33 of the European Parliament.
\end{abstract}

\section{Keywords}

MRI Spine Examinations, Finite Difference Method, Faraday's Equation 


\section{Introduction}

Magnetic Resonance Imaging (MRI) of patients undergoing investigations, are exposed to strong, non-uniform static magnetic fields outside the central imaging region, in which the movement of the body may induce electric currents in tissues which could possibly be harmful [1]. According to Faraday's Law, MRI scans do expose patients to time varying magnetic fields [2], which can induce electrical currents that can cause muscle contraction, nerve stimulation, magnetophosphenes, vertigo, nausea, and metallic taste in the mouth [3].

In Ghana, most patients undergoing MRI investigations are as a result of spinal related injuries. The human spine is one of the delicate organs of the human body because it contains the spinal cord that controls coordination and movement of the human body. As a result, any effect on the spine will have an adverse effect on the body. Hospital data indicate that these injuries may be due to their nature of work-the building industry (carpentry, masonry, laborers), motor mechanics, poor sitting postures, road accidents, carrying of loads on the head, a bad fall, and other such activities. All these activities in one way or the other affect the spine, with the lumbar spine being the most affected because the majority of patients who undergo MRI of the spine complain of lower back pain.

The number of MRI centers in Ghana increased from one in 2008 to ten in 2013. With the increasing number of MRI examinations in Ghana, it is therefore, a quality control measure to ensure that EM field exposure to workers is well below the acceptable maximum limit of $3.8 \mathrm{~V} / \mathrm{m}$ according to EU Directive 2004/40/EC [4], which has since been repealed and replaced with the Directive 2013/35/EU of the European Parliament and of the Council of 26 June 2013 [5]. MR patient exposure is covered by the IEC 60601-2-33 directive [6] and limits are maintained by MR systems and these limits are not to be exceeded. Further, there is the requirement that exposure of workers to radiation does not exceed those of the patients. Therefore, there is the need to further understand and evaluate the effects of EM field exposure to patients undergoing MRI examinations in the local Ghanaian setting. This study was undertaken to re-evaluate established clinical protocols, by estimating the induced EM-fields in patients who underwent MRI spine examination. The study was also to serve as an auditing and additional quality control system for training of MRI technicians and medical physicists in Ghana.

Patient participation was voluntary. The procedures involved in the study were verbally explained to patients using an acceptable language of the patient and their written consent sort. Data were anonymously collected and the procedures met the Ethical and Protocol Review Committees of the 37 Military Hospital, Accra, and Ministry of Health, Accra, Ghana which was approved on 12th September, 2012.

\section{Materials and Methods}

The MRI instrument used was a Philips MR Systems Achieva, (Model No. 
43000-574; PHILIPS, Eindhoven, The Netherlands), with a 1.50 T superconducting magnet. The range of magnetic field strength used for medical imaging is typically from $0.15 \mathrm{~T}$ to $3 \mathrm{~T}$ [7]. In this study, a magnetic field of $1.50 \mathrm{~T}$ was used. The MRI scanner is made up of a long cylindrical hole which is approximately $60 \mathrm{~cm}$ in diameter and open on both ends, a sliding table (couch) which rests in the opening and a sense spine coil $1.50 \mathrm{~T}$ which is placed under the area of the body being examined.

A simulation test was first performed using the mathematical software MATLAB, Mathworks [8] taking into consideration the following factors: mean length, $\boldsymbol{h}$, of the spine $(41.0 \mathrm{~cm}$ was assumed), the mean distance of movement, $\boldsymbol{d}$, (the displacement) and time, $\boldsymbol{t}$, represents the duration of movement of the patient on the couch to the central axis of the MRI scanner. The actual evaluation then extended to cover 120 patients who had been referred for MRI of the spine from October 2012 to January 2013, at the 37 Military Hospital, Accra, Ghana. Patient motion through the MR scanner was recorded using a measuring tape and a stop watch. A combined weight, $\mathrm{kg}$, and height, $\mathrm{m}$, scale was also determined. The results were outputted as means \pm standard deviations (stddev).

\subsection{Model Description and Formulation}

\subsubsection{Field Distribution of Time Varying Magnetic Fields}

A numerical model based on Faraday's equation and experimental data from MRI spine examinations were used to compute the induced EM fields of the human spine. The computation was carried out using the finite difference method (FDM). The FDM is ideally suited for modeling of electromagnetic waves scattering from complex non-canonical objects [9] and MATLAB software.

The computation method was developed as follows:

The expression for a patient's motion, $v$ is

$$
v=\frac{d}{t}
$$

where, $v$ is the velocity of the motion, $d$ is distance and $t$ is time.

Static magnetic fields effects are likely to be caused by the magnetic field vector, $B$, the gradient or the magnetic field, $\frac{\partial B}{\partial z}$, the "force product", $P_{F}$ may be expressed as:

$$
P_{F}=B \frac{\partial B}{\partial z}
$$

where, $z$ is taken as the direction of $B$.

Flow induced electric field, $E_{F}$, depends on the product of the flow velocity, $V$, the magnetic field vector, $B$ and the angle $\theta$ between them, given as:

$$
E_{F}=v B \sin \theta
$$

The motion-induced electric field depends on the geometry and the time rate of change of the magnetic field. 


$$
\oint \underline{E} \cdot \mathrm{d} \underline{l}=-\int_{s} \frac{\mathrm{d} \underline{B}}{\mathrm{~d} t} \cdot \mathrm{d} \underline{s}
$$

The length vector of the body part enclosing the changing magnetic field is $\mathrm{d} l$ and $\mathrm{d} s$ the normal vector to the incremental area $\mathrm{d} A$.

\subsubsection{Transformation of Faraday's Equation}

Transforming the Faraday's equation from Maxwell's time-dependent equations into difference equations with respect to specific field positions on an elementary lattice, the FD technique provides a simple and effective technique for the modeling of a field distribution within the spine of an MRI patient. The system is then solved in a time marching sequence by alternately calculating the electric and magnetic fields in an interlaced spatial grid cell. Given the location of an electromagnetic source and a complete description of the environment in terms of its dielectric parameters; permittivity $(\varepsilon)$, permeability $(\mu)$, conductivity $(\sigma)$, this method provides the ability to assess the electric and magnetic fields.

Therefore, the total magnetic flux passing through a loop is,

$$
\phi_{m}(t)=\int_{s} B \times \mathrm{d} s
$$

Electric potential can also be expressed as;

$$
v(t)=\oint E \times \mathrm{d} l
$$

But from Faraday's law;

$$
v(t)=-\frac{\mathrm{d} \phi_{m}(t)}{\mathrm{d} t}
$$

where, $\phi_{m}(t)$ is the total time varying magnetic flux passing through the surface.

Considering a close cylindrical loop, substituting Equation (5) into Equation (7),

$$
\begin{array}{r}
v(t)=-\int \frac{B}{\mathrm{~d} t} \times \mathrm{d} s \\
v(t)=-\frac{\mathrm{d}}{\mathrm{d} t} \int B \times \mathrm{d} s
\end{array}
$$

where, $\mathrm{d} s=\operatorname{Area}(A)$.

The differential equation of

$$
\frac{\partial^{2} A}{\partial r^{2}}=2 \pi r^{2} h
$$

Therefore, Equation (9) becomes

$$
\begin{aligned}
v(t) & =-\frac{\mathrm{d}}{\mathrm{d} t} \int B \times A \\
& =-\frac{\partial B}{\partial t} \times \frac{\partial A}{\partial t}
\end{aligned}
$$

From Equations (6) and (9) 


$$
\oint E \times \mathrm{d} l=-\frac{\mathrm{d}}{\mathrm{d} t} \int B \times \mathrm{d} s
$$

Equation (11) is the integral form of the Faraday's Law.

Using Stokes' theorem [10] to transform the line integral into a surface integral;

$$
\oint \underline{E} \times \mathrm{d} l=\int_{s}(\underline{\nabla} \times \underline{E}) \times \mathrm{d} a
$$

Therefore, the differential form of the Faraday's law of induction is;

$$
\begin{aligned}
& \underline{\nabla} \times \underline{E}=-\frac{\partial \underline{B}}{\partial t} \\
\underline{\nabla} \times \underline{E}= & \left(\frac{1}{r} \frac{\partial E_{z}}{\partial \theta}-\frac{\partial E_{\theta}}{\partial z}\right) \hat{r}+\left(\frac{\partial E_{r}}{\partial z}-\frac{\partial E_{z}}{\partial r}\right) \hat{\theta} \\
+ & \frac{1}{r}\left(\frac{\partial}{\partial r}\left(r E_{\theta}\right)-\frac{\partial E_{r}}{\partial \theta}\right) \hat{z}
\end{aligned}
$$

Note that the determinant;

$$
\underline{\nabla} \times \underline{E}=\left[\begin{array}{ccc}
\hat{r} & r \hat{\theta} & \hat{z} \\
\frac{\partial}{\partial r} & \frac{\partial}{\partial \theta} & \frac{\partial}{\partial z} \\
E_{r} & r E \theta & E_{z}
\end{array}\right]
$$

Formulating EM wave equation of the spine from Maxwell's equation;

$$
\begin{gathered}
\nabla \times B=\phi \nabla \times E=0 \\
\underline{\nabla} \times \underline{E}=-\frac{\partial \underline{B}}{\partial t} \underline{\nabla} \times \underline{B}=\varepsilon_{\circ} \mu_{\circ} \frac{\partial \underline{E}}{\partial t} \\
\underline{\nabla} \times \underline{\nabla} \times \underline{E}=-\underline{\nabla} \times \frac{\partial \underline{B}}{\partial t} \\
\underline{\nabla} \times \underline{\nabla} \times \underline{E}=\underline{\nabla}(\underline{\nabla} \cdot E)-\underline{\nabla}^{2} \underline{E} \\
-\frac{\partial}{\partial t} \underline{\nabla} \times \underline{\nabla}=\underline{\nabla}(\underline{\nabla} \cdot \underline{\nabla})-\underline{\nabla^{2}} \underline{E} \\
-\frac{\partial \underline{B}}{\partial t} \underline{\nabla} \times \underline{B}=\varepsilon_{\circ} \mu_{\circ} \frac{\partial^{2} \underline{E}}{\partial t^{2}}
\end{gathered}
$$

Combing Equations (19) and (20);

$$
\underline{\nabla}(\underline{\nabla} \times \underline{\nabla})-\underline{\nabla}^{2} \underline{E}=-\varepsilon_{o} \mu_{o} \frac{\partial^{2} \underline{E}}{\partial t^{2}}
$$

where, $\nabla(\nabla \times E)=0$

$$
-\underline{\nabla}^{2} \underline{E}=-\varepsilon_{o} \mu_{o} \frac{\partial^{2} \underline{E}}{\partial t^{2}}
$$

Equation (22) indicates the modified Maxwell's equation, where, $\varepsilon_{\circ} \mu_{\circ}=\frac{1}{v^{2}}$.

Therefore, Equation (22) becomes 


$$
-\underline{\nabla} \underline{E}=-\frac{1}{v^{2}} \frac{\partial^{2} E}{\partial t^{2}}
$$

Since the EM field is polarized in the y direction, the induced EM field in the 3D plane within the spine becomes;

$$
\begin{aligned}
& \text { From Equation (14) } \phi_{m}(t)=\int_{s} B \times \mathrm{d} s \\
& \qquad \frac{\partial^{2} \phi_{m}}{\partial r^{2}}+\frac{\partial^{2} \phi_{m}}{\partial z^{2}}=\frac{1}{2 \pi r h} \frac{\partial^{2} \phi_{m}}{\partial t^{2}}
\end{aligned}
$$

The discretization of Equation (25) yields the EM field within various positions of the spine.

$$
2 \pi h\left(\frac{\partial^{2} \phi_{m}}{\partial r^{2}}+\frac{\partial^{2} \phi_{m}}{\partial z^{2}}\right)=\frac{\partial^{2} \phi_{m}}{\partial t^{2}}
$$

where, $\boldsymbol{h}$ is the length of the spine.

\subsubsection{Discretization of Spine Model}

The discretization of the partial differential equation, Equation (25) is illustrated in Figure 1, giving rise to the solution, Equation (26), where, $i, j$ and $k$ represent variables temperature, length and time, respectively.

$$
\phi_{i, j, k}=\frac{\sum_{m=0}^{1}\left(\phi_{i-m, j, k}-2 \phi_{i, j, k}+\phi_{i+m, j, k}\right)}{\Delta r^{2}}+\frac{\sum_{m=0}^{1}\left(\phi_{i, j-m, k}-2 \phi_{i, j, k}+\phi_{i, j+1, k}\right)}{\Delta z^{2}}
$$

Therefore, the final discretized EM-field equation from the Faraday's equation becomes;

$\mathrm{i}, \quad \mathrm{j}+1$

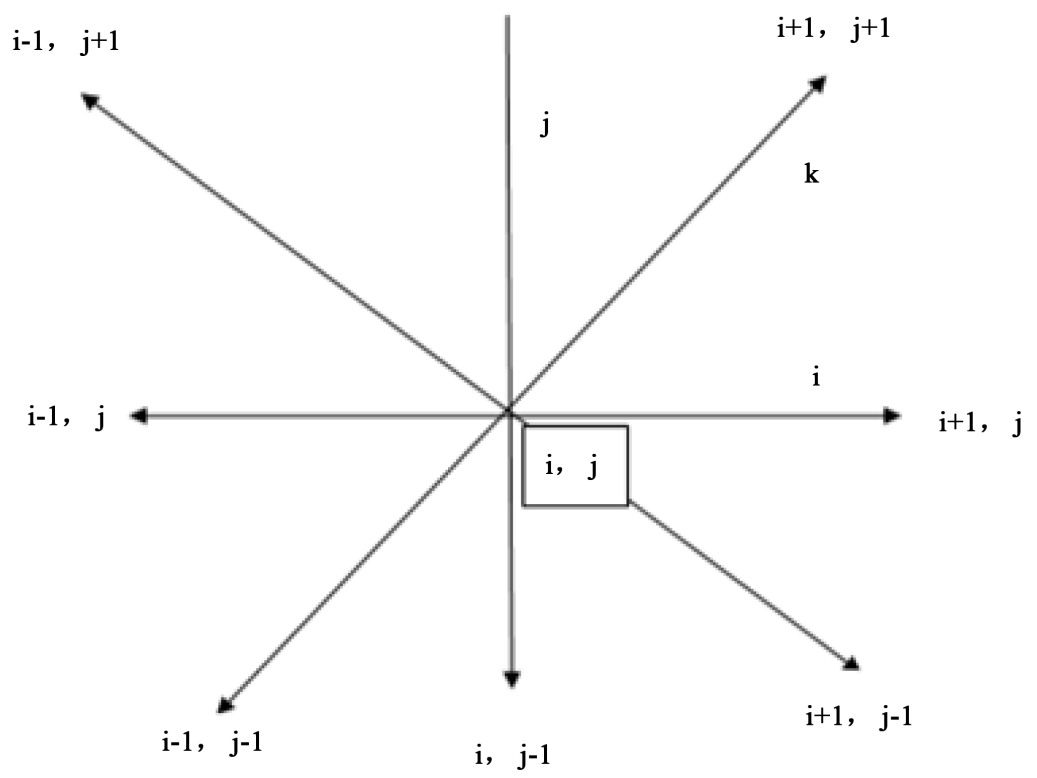

Figure 1. The discretization of the partial differential Equation (25) into discrete counterparts. 


$$
\Rightarrow \frac{\phi_{i, j}^{n+1}-2 \phi_{i, j}^{n}+\phi_{i, j}^{n-1}}{\left(\Delta t^{2}\right)}=v^{2} \omega\left[\frac{\phi_{i+1, j}^{n}-2 \phi_{i, j}^{n}+\phi_{i-1, j}^{n}}{(\Delta h)^{2}}+\frac{\phi_{i, j+1}^{n}-2 \phi_{i, j}^{n}+\phi_{i, j-1}^{n}}{(\Delta h)^{2}}\right]
$$

where, $\phi^{n}, \phi^{n+1}$ represent the iterated EM fields within the spatial grids of the spine and $\Delta h$ is the step size.

\section{Results and Discussions}

\subsection{Simulation Results}

The induced EM fields were computed for the three sections of the spine as the patients underwent the MRI examinations using FDM and MATLAB. The simulated results of the induced electric field $(0.39 \mathrm{~V} / \mathrm{m})$ and current density $\left(0.039 \mathrm{~A} / \mathrm{m}^{2}\right)$ were lower than the measured results $\left(0.44 \mathrm{~V} / \mathrm{m}\right.$ and $0.043 \mathrm{~A} / \mathrm{m}^{2}$, respectively) and this was due to the fact that during the simulation an assumed length of the spine $(41.0 \mathrm{~cm})$ was assumed for all patients.

\subsection{Experimental Results}

A total of 120 patients were MRI examined of which 75 patients ranging in ages from 8 to 90 years underwent lumbar spine examinations (Table 1). The study revealed that most of the patients undergoing the lumbar spine examination were mainly drivers, civil servants, artisans, and traders, whose activities involve several hours of sitting or standing for extended periods (Table 1). There were a few cases in which the patients were involved in motor accidents. The lumbar spine had the largest displacement with a mean ( \pm stddev) value of $1.46 \pm 0.06 \mathrm{~m}$ compared with the thoracic spine, $1.33 \pm 0.36 \mathrm{~m}$ and the cervical spine, $1.10 \pm$ $0.10 \mathrm{~m}$ (Table 2).

The lumbar spine had the highest duration with a mean of $5.98 \pm 0.48 \mathrm{~s} \mathrm{com}$ pared with the cervical spine, $5.92 \pm 0.40 \mathrm{~s}$ and the thoracic spine, $5.88 \pm 0.67 \mathrm{~s}$.

Table 1. Gender, age range and number of patients for the various spine examinations.

\begin{tabular}{cccc}
\hline Gender & Cervical & Thoracic & Lumbar \\
\hline Male & 20 & 7 & 44 \\
Female & 10 & 8 & 31 \\
Total & 30 & 15 & 75 \\
Mean age (years) & $51.22 \pm 15.71$ & $43.87 \pm 19.85$ & $51.88 \pm 15.06$ \\
Mean height $(\mathrm{m})$ & $1.63 \pm 0.13$ & $1.62 \pm 0.14$ & $1.65 \pm 0.11$ \\
Mean weight $(\mathrm{kg})$ & $75.60 \pm 19.89$ & $71.40 \pm 15.37$ & $76.40 \pm 12.19$ \\
\hline
\end{tabular}

Table 2. Results of distance and time measurements.

\begin{tabular}{cccc}
\hline Parameters & Cervical & Thoracic & Lumbar \\
\hline Mean Distance (m) & $1.10 \pm 0.10$ & $1.33 \pm 0.36$ & $1.46 \pm 0.06$ \\
Mean Time (s) & $5.92 \pm 0.40$ & $5.88 \pm 0.67$ & $5.98 \pm 0.48$ \\
\hline
\end{tabular}


The results showed that, a maximum velocity of $0.29 \mathrm{~m} / \mathrm{s}$, with peak induced electric field and current density of $0.44 \mathrm{~V} / \mathrm{m}$ and of $0.043 \mathrm{~A} / \mathrm{m}^{2}$ were recorded, respectively. The results of the induced electric field were compared to the limits given in the directive IEC 60601-2-33 standard [6]. The comparison shows that the derived peak electric field of $0.44 \mathrm{~V} / \mathrm{m}$ was much lower than the IEC standard of $3.8 \mathrm{~V} / \mathrm{m}$ in the normal operating mode and $4.7 \mathrm{~V} / \mathrm{m}$ in the first level operating mode. These values are lower than the onset thresholds of $6-7 \mathrm{~V} / \mathrm{m}$ for peripheral nerve stimulation (PNS). According to Directive 2013/35/EU [5] for EMF, exposure limit of current density for the head and torso is $3 \mathrm{~A} / \mathrm{m}^{2}$. The result of the induced current densities in the spinal cord was $0.043 \mathrm{~A} / \mathrm{m}^{2}$ which as much lower than the limit of $3 \mathrm{~A} / \mathrm{m}^{2}$. Similarly, the induced current densities in the skin and spine were lower than the Directive 2013/35/EU exposure limit [5].

Solutions to the discretized partial differential equations obtained with the finite difference method showing the variations in induced EM fields for male (Figure 2 and Figure 3) and female (Figure 4 and Figure 5) patients, who underwent the MRI examination of the lumbar spine are displayed in two-dimension (2D) and three-dimension (3D). For the 2D profiles for males (Figure 2), the mean induced EM field of $0.24 \mathrm{~V} / \mathrm{m}$ (range 0.0 to $0.38 \mathrm{~V} / \mathrm{m}$ ) was recorded while the female profile (Figure 4), recorded a mean value of $0.22 \mathrm{~V} / \mathrm{m}$ and ranges from 0.0 to $0.36 \mathrm{~V} / \mathrm{m}$. For the 3D profiles, the induced EM fields were static with time, $\boldsymbol{t}$ and varied with position, $\boldsymbol{r}$. For both the 3D profiles, concentration of the induced EM fields was evenly distributed which depicts a hyperbolic shape. The color maps represent the concentrations of the EM fields; the blue color represents low EM field region whiles the red color represents high EM field region. Comparing the 3D profiles for both genders, the female lumbar (Figure 5) was more highly concentrated than the male profile (Figure 3).

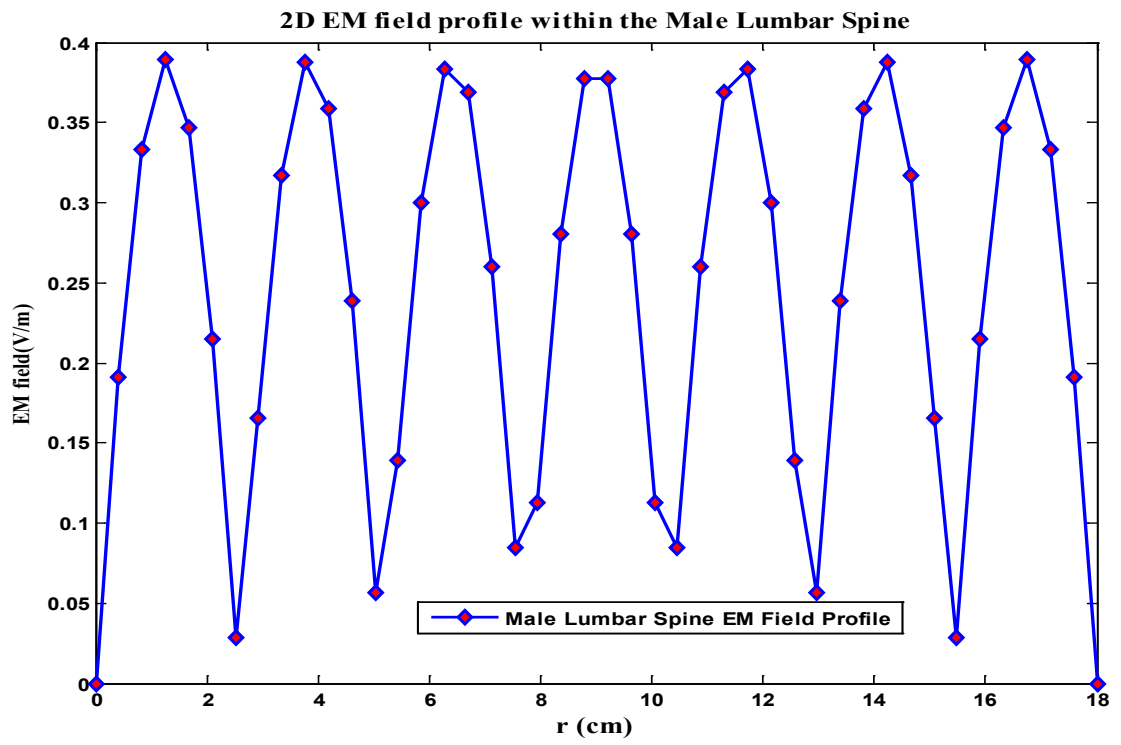

Figure 2. Variations in induced EM-Field for male lumbar spine in 2D. 


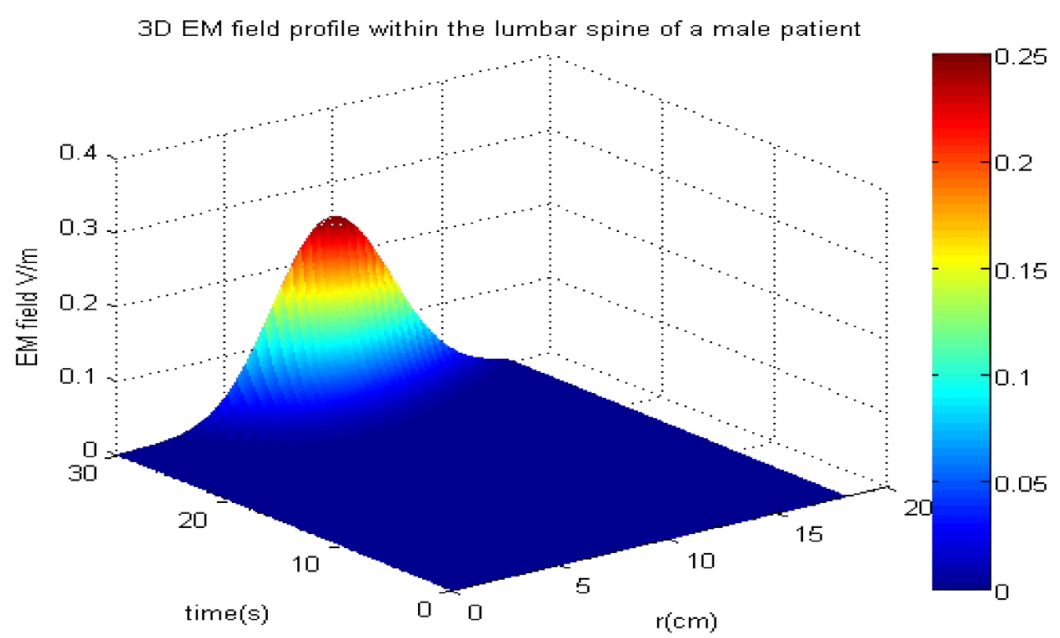

Figure 3. Variations in induced EM-Field for male lumbar spine in 3D profiles.

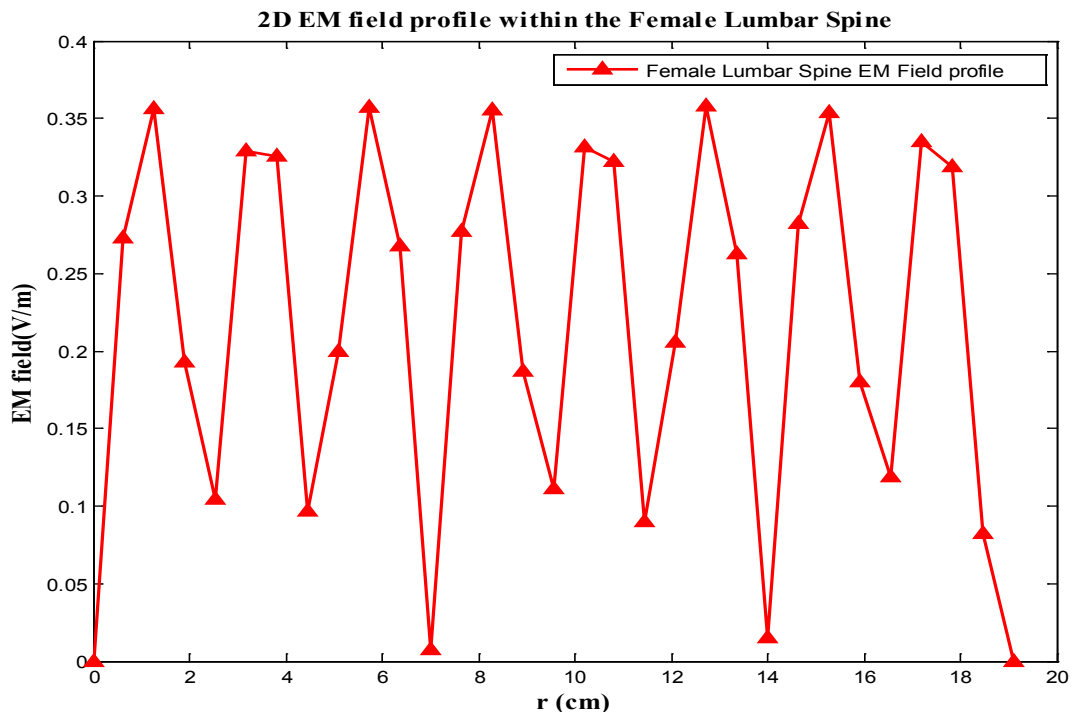

Figure 4. Variations in induced EM-Field for female lumbar spine in 2D profiles.

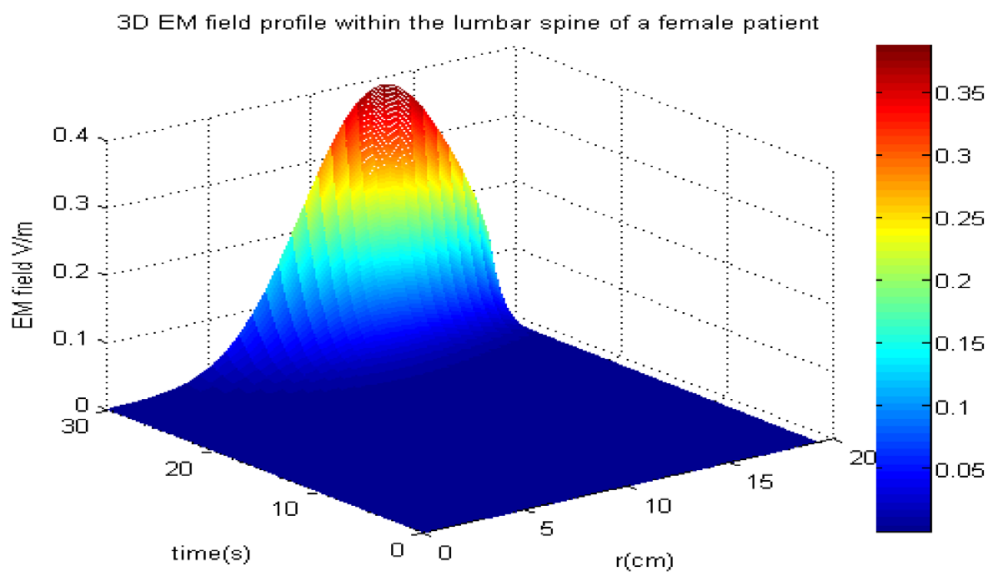

Figure 5. Variations in induced EM-Field for female lumbar spine in 3D profiles. 
From this study, the simulated results of the induced electric field $(0.39 \mathrm{~V} / \mathrm{m})$ and current density $\left(0.039 \mathrm{~A} / \mathrm{m}^{2}\right)$ the experimental results; induced electric field $(0.44 \mathrm{~V} / \mathrm{m})$ and current density $\left(0.043 \mathrm{~A} / \mathrm{m}^{2}\right)$ were much lower compared with the report by Liu et al. [1], where the simulated results of the induced electric field and current density $\left(1.8 \mathrm{~V} / \mathrm{m}\right.$ and $0.22 \mathrm{~A} / \mathrm{m}^{2}$ respectively) and the experimental results $\left(1.8 \mathrm{~V} / \mathrm{m}\right.$ and $0.21 \mathrm{~A} / \mathrm{m}^{2}$ respectively). Here, a lower magnetic field strength of $1.5 \mathrm{~T}$ was used as compared with the $4.0 \mathrm{~T}$ used for simulation, with the maximum current of about $220 \mathrm{~mA} / \mathrm{m}^{2}$ in the report by Liu et al., [1]. In modern MRI scanners, Faraday currents can reach a value of $386 \mathrm{~mA} / \mathrm{m}^{2}$ [11]. Further, Bowtell and Bowley [12], indicated that, the electric field peak value of about $1.8 \mathrm{~V} / \mathrm{m}$ (for the $4 \mathrm{~T}$ MRI scanner, with a corresponding duration of movement of $0.5 \mathrm{~m} / \mathrm{s}$ movement), is much lower than $6.2 \mathrm{~V} / \mathrm{m}$, which has been suggested to be the threshold for nerve stimulation at low frequencies [5].

\section{Conclusion}

The results of the level of induced EM-fields were such that they might not pose any potential health hazards to the patients as these values were well below the recommended guidance levels set by Directives 2013/35/EU [5] and IEC 60601-2-33 standards [6] exposure limits for workers and patients, respectively. We also recommend periodic reviews of such protocols of newer NMR instruments to help shape safety policies for both patients and workers.

\section{Conflict of Interest}

The authors declare that there are no conflicts of interests regarding the study and publication of this paper.

\section{Acknowledgements}

We would like to express our sincere thanks to Prof. AWK Kyere, Head of Medical Physics Department, Graduate School of Nuclear and Allied Sciences, Legon, Ghana, the staff of the MRI Unit at 37 Military Hospital and Ghana Standards Authority.

\section{References}

[1] Liu, F., Zhao, H. and Crozier, S. (2003) Calculation of the Electric Fields Induced by Body and Head in High-Field MRI. Journal of Magnetic Resonance, 161, 99-107. http://pfeifer.phas.ubc.ca/refbase/files/Liu-JMagRes-2003-161-99.pdf https://doi.org/10.1016/S1090-7807(02)00180-5

[2] Mcrobbie, D.W. (2012) Occupational Exposure in MRI. British Journal of Radiology, 85, 293-312. https://www.ncbi.nlm.nih.gov/pmc/articles/PMC3486652/ https://doi.org/10.1259/bjr/30146162

[3] ICNIRP (2014) Guidelines for Limiting Exposure to Electric Fields Induced by Movement of the Human Body in a Static Magnetic Field and by Time-Varying Magnetic Fields below $1 \mathrm{~Hz}$. Health Physics, 106, 418-425. https://www.icnirp.org/cms/upload/publications/ICNIRPmvtgdl_2014.pdf 
https://doi.org/10.1097/HP.0b013e31829e5580

[4] Directive 2004/40/EC of the European Parliament and of the Council (2004). Official Journal of the European Union, L184/1, L184-9.

https://archive.senseaboutscience.org/data/files/PhysicalAgentsDirective.pdf

[5] Directive 2013/35/EU-Electromagnetic Fields.

https://osha.europa.eu/en/legislation/directives/directive-2013-35-eu-electromagnet ic-fields

[6] IEC 60601-2-33:2010+AMD1:2013+AMD2:2015 CSV (2010) Medical Electrical Equipment-Part 2-33: Particular Requirements for the Basic Safety and Essential Performance of Magnetic Resonance Equipment for Medical Diagnosis. Consolidated Version, 2010, 2013, 2015.

https://webstore.iec.ch/preview/info_iec60601-2-33\%7Bed3.0\%7Db.pdf

[7] MR-TIP (2012) MRI. http://www.mr-tip.org/mri

[8] MATLAB (2013) Mathworks. http://mathworks.com/products/matlab

[9] Taflove, A. and Hagness, S.C. (2005) Computational Electrodynamics: The Finite-Difference Time-Domain Method. 3rd Edition, Artech House, Norwood, MA.

[10] Green, P. and Rosenberg, J. (2010) Line Integrals around Closed Curves, and the Theorems of Green and Stokes.

https://qmplus.qmul.ac.uk/pluginfile.php/283380/mod_resource/content/3/Chapter \%204\%20-\%20Vector\%20intergration\%2C\%202010.pdf

[11] Schmitt, F., Stehling, M.K. and Turner, R. (1998) Echo-Planar Imaging Theory, Technique and Application. Springer, New York, 9-14.

http://radioramanuja.com/file/download/echo-planar-imaging-theory-technique-an d-application

https://doi.org/10.1007/978-3-642-80443-4

[12] Bowtell, R. and Bowley, R.M. (2000) Analytic Calculations of the E-Fields Induced by Time-Varying Magnetic Fields Generated by Cylindrical Gradient Coils. Magnetic Resonance in Medicine, 44, 782-790.

https://doi.org/10.1002/1522-2594(200011)44:5<782::AID-MRM16>3.0.CO;2-7 